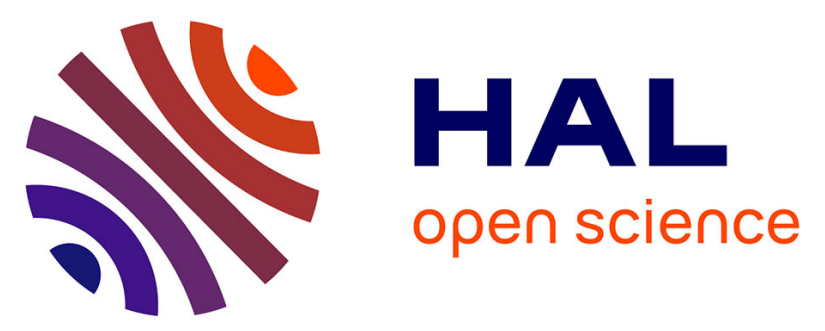

\title{
High expression of the RNA-binding protein RBPMS2 in gastrointestinal stromal tumors
}

Ilona Hapkova, Josef Skarda, Caroline Rouleau, An Thys, Cécile Notarnicola, Maria Janikova, Florence Bernex, Miroslav Rypka, Jean-Marie Vanderwinden, Sandrine Faure, et al.

\section{To cite this version:}

Ilona Hapkova, Josef Skarda, Caroline Rouleau, An Thys, Cécile Notarnicola, et al.. High expression of the RNA-binding protein RBPMS2 in gastrointestinal stromal tumors. Experimental and Molecular Pathology, 2013, 10.1016/j.yexmp.2012.12.004 . hal-02543718

\section{HAL Id: hal-02543718 \\ https://hal.umontpellier.fr/hal-02543718}

Submitted on 15 Apr 2020

HAL is a multi-disciplinary open access archive for the deposit and dissemination of scientific research documents, whether they are published or not. The documents may come from teaching and research institutions in France or abroad, or from public or private research centers.
L'archive ouverte pluridisciplinaire HAL, est destinée au dépôt et à la diffusion de documents scientifiques de niveau recherche, publiés ou non, émanant des établissements d'enseignement et de recherche français ou étrangers, des laboratoires publics ou privés. 


\title{
High expression of the RNA-binding protein RBPMS2 in gastrointestinal stromal tumors
}

\author{
Ilona Hapkova ${ }^{\text {a,b }}$, Josef Skarda ${ }^{c}$, Caroline Rouleau ${ }^{\mathrm{a}, \mathrm{d}}$, An Thys ${ }^{\mathrm{e}}$, Cécile Notarnicola a, Maria Janikova ${ }^{\mathrm{c}}$, \\ Florence Bernex ${ }^{\text {a }}$, Miroslav Rypka ${ }^{\mathrm{b}}$, Jean-Marie Vanderwinden ${ }^{\mathrm{e}}$, Sandrine Faure ${ }^{\mathrm{a}}$, \\ Jaroslav Vesely ${ }^{\mathrm{b}}$, Pascal de Santa Barbara ${ }^{\mathrm{a}, *}$ \\ a INSERM U1046, Université Montpellier 1, Université Montpellier 2, Montpellier, France \\ ${ }^{\mathrm{b}}$ Department of Pathophysiology, Faculty of Medicine and Dentistry, Palacky University, Olomouc, Czech Republic \\ c Department of Clinical and Molecular Pathology, Faculty of Medicine and Dentistry, Palacky University and University Hospital, Olomouc, Czech Republic \\ d CHU Montpellier, Service d'Anatomie Pathologique, Montpellier, France \\ e Laboratory of Neurophysiology, Faculty of Medecine, Université Libre de Bruxelles, Brussels, Belgium
}

\section{A R T I C L E I N F O}

Keywords:

Gastrointestinal stromal tumors (GIST)

RNA-binding protein

RBPMS2

KIT

Smooth muscle cells

Interstitial cell of Cajal

\begin{abstract}
A B S T R A C T
Gastrointestinal stromal tumors (GISTs) are the most common mesenchymal neoplasms of the gastrointestinal tract and are often associated with KIT or PDGFRA gene mutations. GIST cells might arise from the interstitial cells of Cajal (ICCs) or from a mesenchymal precursor that is common to ICCs and smooth muscle cells (SMCs). Here, we analyzed the mRNA and protein expression of RNA-Binding Protein with Multiple Splicing-2 (RBPMS2), an early marker of gastrointestinal SMC precursors, in human GISTs $(n=23)$ by in situ hybridization, quantitative RT-PCR analysis and immunohistochemistry. The mean RBPMS2 mRNA level in GISTs was 42-fold higher than in control gastrointestinal samples $(p<0.001)$. RBPMS2 expression was not correlated with KIT and PDGFRA expression levels, but was higher in GISTs harboring KIT mutations than in tumors with wild type KIT and PDGFRA or in GISTs with PDGFRA mutations that were characterized by the lowest RBPMS2 levels. Moreover, RBPMS2 levels were 64-fold higher in GIST samples with high risk of aggressive behavior than in adult control gastrointestinal samples and 6.2-fold higher in high risk than in low risk GIST specimens. RBPMS2 protein level was high in $87 \%$ of the studied GISTs independently of their histological classification. Finally, by inhibiting the KIT signaling pathway in GIST882 cells, we show that RBPMS2 expression is independent of KIT activation. In conclusion, RBPMS2 is up-regulated in GISTs compared to normal adult gastrointestinal tissues, indicating that RBPMS2 might represent a new diagnostic marker for GISTs and a potential target for cancer therapy.
\end{abstract}

\section{Introduction}

Gastrointestinal stromal tumors (GISTs) are the most common mesenchymal neoplasms of the gastrointestinal tract (Corless et al., 2011) and are highly resistant to conventional chemotherapy and radiotherapy. These tumors are characterized by the presence of activating mutations in KIT (75-80\% frequency) or Platelet-Derived Growth Factor Receptor Alpha (PDGFRA) (5-10\% of tumors), two genes encoding receptors for growth factors that are normally activated only in specific situations (Hirota et al., 1998). Imatinib mesylate, a small-molecular tyrosine kinase inhibitor that targets phosphorylation/activation of KIT and PDGFRA and also constitutively activated KIT and PDGFRA proteins, has proven efficient in GIST treatment (Joensuu et al., 2001; Tuveson et

\footnotetext{
* Corresponding author at: INSERM U1046, 371 Avenue Doyen Giraud, 34295 Montpellier, France. Fax: + 33467415231.

E-mail address: Pascal.de-Santa-Barbara@inserm.fr (P. de Santa Barbara).
}

al., 2001); however, resistance to such therapy is increasing. Therefore, the development of new-targeted therapies is strongly encouraged (Renouf et al., 2009).

It is now largely documented that post-regulatory RNA events play crucial roles in modulating differentiation and remodeling of smooth muscle tissues (Notarnicola et al., 2012; Xin et al., 2009). RNA-protein complexes control multiple steps of this process, including mRNA cellular localization, splicing, translational regulation and degradation (St Johnston, 2005). For instance, the expression of an alternative splicing isoform of the natural killer (NK) cell receptor NKp30 correlates with the prognosis of GISTs, independently from the KIT mutations (Delahaye et al., 2011).

Moreover, RNA-binding proteins (RBPs), which play important roles in regulating RNA metabolism, may also be deregulated in diseases, particularly in cancers during the initiation and progression phases (van Kouwenhove et al., 2011). The RNA Recognition Motif (RRM) proteins form a large RBP family which includes also RNABinding Protein for Multiple Splicing-2 (RBPMS2), an early marker 
Table 1

Primers used for quantitative RT-PCR amplification of selected human genes.

\begin{tabular}{lll}
\hline$\frac{\text { Target }}{\text { transcript }}$ & Forward primer & $\frac{\text { Reverse primer }}{\left(5^{\prime}-3^{\prime}\right)}$ \\
\cline { 2 - 3 } KIT & $\left(5^{\prime}-3^{\prime}\right)$ & AGG AAG TTG TGT TGG GTC TA \\
TMEM16A & CCT TTG CTG ATT GGT TTC G & TCG TCG GCA TCT TCA GT \\
RBPMS2 & GCT TCC GCA GGG AGG AGT A & GGG TGG TGT CAG AGG AAG \\
PDGFRA & CTC CCA TGC TGC GTT CA & CCC TCA CTG TTG TGT AAG GTT \\
HPRT & ATG TGC CAG ACC CAG AT & TGA CCA AGG AAA GCA AAG TCT G \\
Calponin & TGT AAT GAC CAG TCA ACA GGG & CAG CCC AAT GAT GTT CCG \\
$\alpha$ SMA & AGA AGT ATG ACC ACC AGC & GAA GGA ATA GCC ACG CTC AG \\
Desmin & TTC AAT GTC CCA GCC ATG TA & TTT GCT CAG GGC TGG TTT \\
SM22 & AGG AGA TGA TGG AAT ACC GAC & CTG TTG CTG CCC ATC TGA AG \\
PCNA & GCA GTC CAA AAT CGA GAA GA & GGT GTC GAA GCC CTC AG \\
\hline
\end{tabular}

of gastrointestinal smooth muscle precursor cells that we identified recently (Notarnicola et al., 2012). We showed that ectopic expression of RBPMS2 in differentiated SMCs hinders their ability to contract, favors their proliferation and leads to their dedifferentiation, demonstrating that RBPMS2 expression must be tightly regulated to avoid SMC dedifferentiation (Notarnicola et al., 2012).

These results and the fact that GISTs are thought to arise from Interstitial Cells of Cajal (ICCs) or from a mesenchymal precursor that is common to ICCs and smooth muscle cells (SMCs) (Faure and de Santa Barbara, 2011; Le Guen et al., 2009; Sanders et al., 2006) prompted us to examine the mRNA and protein expression of RBPMS2 in different categories of human adult GISTs and in the GIST882 cell line. We found that RBPMS2 is up-regulated in GISTs compared to normal adult gastrointestinal tissues.

\section{Materials and methods}

Tumor samples and KIT and PDGFRA mutational analysis

Paraffin-embedded tumor samples from primary GISTs of 23 adult patients before Imatinib treatment were collected in the Department of Clinical and Molecular Pathology of the Olomouc University Hospital (Olomouc, Czech Republic). The risk of clinically aggressive behavior was evaluated according to the consensus approach published by Fletcher and coworkers (Fletcher et al., 2002). Control samples were normal gastrointestinal tissue specimens isolated from adult epithelialderived tumors. For KIT and PDGFRA mutational analysis, genomic DNA was extracted from the paraffin-embedded GIST samples using the QIAamp DNA FFPE Tissue Kit (Qiagen). Specific PCR primers were used
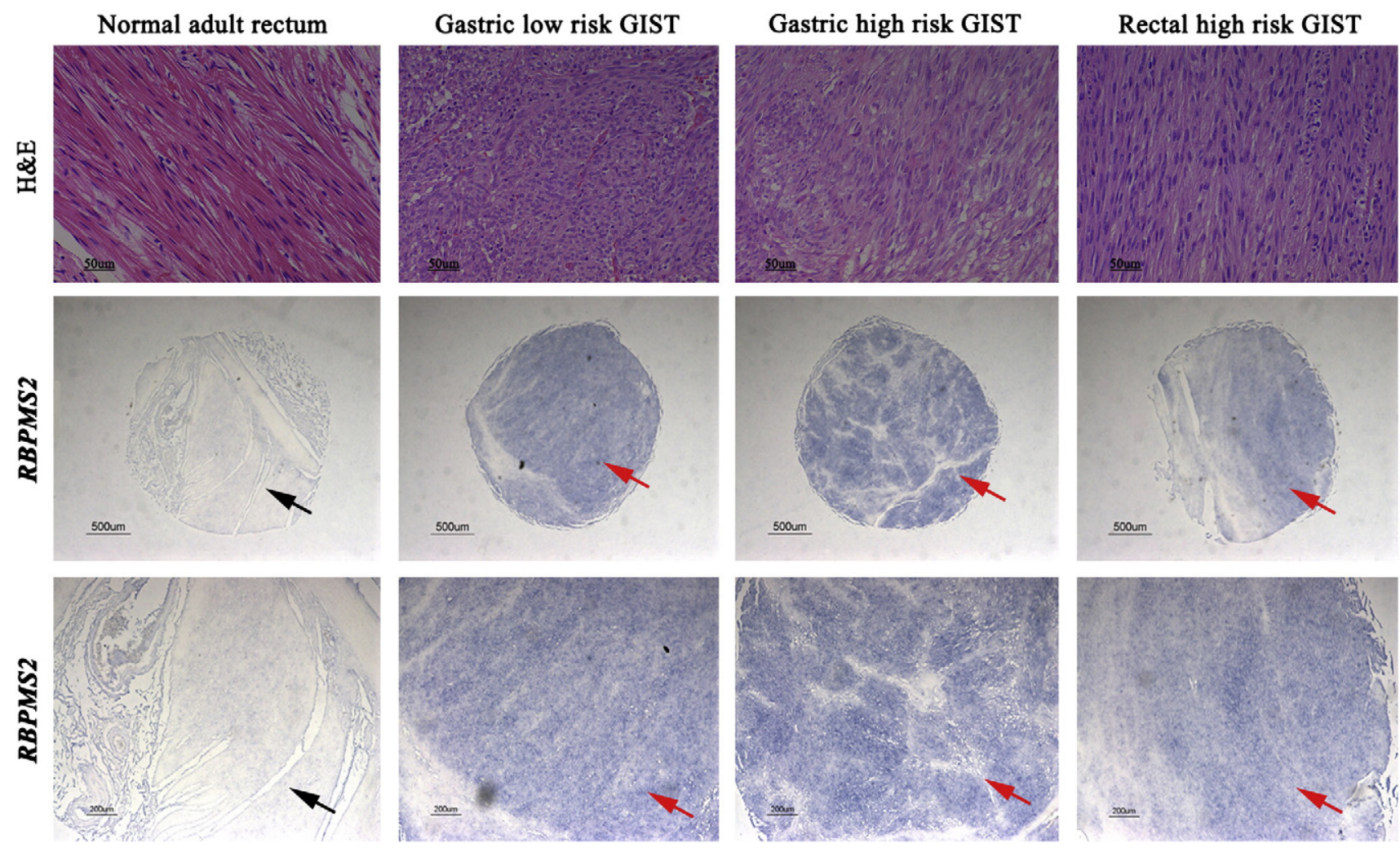

Fig. 1. Expression of RBPMS2 transcripts in adult normal rectum and GIST samples. Hematoxylin-eosin (H\&E) staining (upper panels) and in situ hybridization of smooth muscle layers sections from normal rectum and GIST samples (middle and lower panels show different magnifications of the same sections) with an anti-human RBPMS2 riboprobe. RBPMS2 transcripts are not detected in adult normal rectal musculature (arrows), whereas they are strongly expressed in tumor cells (red arrows). Bars, 50 um (upper panels), $500 \mu \mathrm{m}$ (middle panels) and $200 \mu \mathrm{m}$ (lower panels). 
Table 2

Summary of the clinical and morphological features, risk assessment, KIT and PDGFRA mutational status as well as KIT and RBPMS2 expressions in the GIST cohort.

\begin{tabular}{|c|c|c|c|c|c|c|c|c|c|c|}
\hline Patient & Sex & $\begin{array}{l}\text { Age } \\
\text { (years) }\end{array}$ & Site & Morphology & Size $(\mathrm{mm})$ & Risk & KIT or PDGFRA mutations & KIT RT-PCR & $\begin{array}{l}\text { RBPMS2 } \\
\text { RT-PCR }\end{array}$ & $\begin{array}{l}\text { RBPMS2 } \\
\text { IHC }\end{array}$ \\
\hline 1 & M & 73 & Small intestine & Spindle & $90 \times 100 \times 110$ & High Risk & Exon 9 ins AY502-503 KIT & +++ & +++ & ++ \\
\hline 2 & M & 73 & Soft tissue & Epithelioid & $12 \times 5 \times 12$ & High Risk & Exon 9 ins AY502-503 KIT & +++ & +++ & +++ \\
\hline 3 & M & 74 & Duodenum & Spindle & $70 \times 75 \times 85$ & High Risk & Exon 9 ins AY502-503 KIT & ++ & ++ & +++ \\
\hline 4 & M & 71 & Small intestine & Spindle & $14 \times 5 \times 14$ & Low Risk & Exon 11 V559D KIT & ++ & +++ & +++ \\
\hline 5 & $\mathrm{M}$ & 60 & Rectum & Spindle & $35 \times 20 \times 15$ & Low Risk & Exon 11 V559D KIT & - & ++ & ++ \\
\hline 6 & M & 71 & Stomach & Mixed & $15 \times 12 \times 10$ & High Risk & Exon $11 \mathrm{del} / \mathrm{ins}$ KIT & +++ & +++ & + \\
\hline 7 & M & 77 & Esophagus & Spindle & $22 \times 18 \times 40$ & High Risk & Exon $11 \mathrm{del} /$ ins KIT & +++ & +++ & ++ \\
\hline 8 & M & 74 & Rectum & Spindle & $80 \times 75 \times 60$ & Low Risk & Exon $11 \mathrm{del} /$ ins KIT & + & +++ & ++ \\
\hline 9 & M & 66 & Stomach & Spindle & $9 \times 5 \times 5$ & Low Risk & Exon 13 K642E KIT & ++ & +++ & NA \\
\hline 10 & $\mathrm{~F}$ & 67 & Stomach & Epithelioid & $43 \times 47 \times 35$ & High Risk & E12 V561D PDGFRA & + & ++ & ++ \\
\hline 11 & $\mathrm{~F}$ & 62 & Stomach & Mixed & NA & Low Risk & Exon 18 D842Y PDGFRA & ++ & +++ & NA \\
\hline 12 & $\mathrm{~F}$ & 58 & Colon & Mixed & $10 \times 10 \times 10$ & Low Risk & Exon 18 silent mutation V824V PDGFRA & ++ & ++ & ++ \\
\hline 13 & M & 36 & Recto-peritoneum & Mixed & $65 \times 45 \times 42$ & Low Risk & Exon 18 silent mutation V824V PDGFRA & + & ++ & + \\
\hline 14 & $\mathrm{~F}$ & 70 & Esophagus & Mixed & $50 \times 30 \times 30$ & Low Risk & WT & +++ & +++ & ++ \\
\hline 15 & M & 48 & Esophagus & Spindle & $35 \times 25 \times 20$ & High Risk & WT & + & + & ++ \\
\hline 16 & M & 61 & Stomach & Spindle & $45 \times 25 \times 10$ & Low Risk & WT & ++ & +++ & NA \\
\hline 17 & M & 42 & Small intestine & Mixed & $40 \times 30 \times 20$ & High Risk & WT & +++ & +++ & + \\
\hline 18 & M & 60 & Duodenum & Mixed & $40 \times 32 \times 22$ & Low Risk & WT & ++ & +++ & + \\
\hline 19 & M & 81 & Rectum & Mixed & $1 \times 3 \times 15$ & High Risk & WT & + & ++ & ++ \\
\hline 20 & $\mathrm{~F}$ & 55 & Recto-peritoneum & Epithelioid & $90 \times 70 \times 50$ & High Risk & WT & +++ & +++ & ++ \\
\hline 21 & M & 65 & Small intestine & Epithelioid & $12 \times 8 \times 8$ & High Risk & NA & NA & NA & ++ \\
\hline 22 & $\mathrm{~F}$ & 55 & Small intestine & Spindle & $150 \times 13 \times 110$ & High Risk & NA & NA & NA & + \\
\hline 23 & $\mathrm{~F}$ & 60 & Stomach & Spindle & $50 \times 40 \times 50$ & High Risk & Exon $11 \mathrm{del} /$ ins KIT & NA & NA & + \\
\hline
\end{tabular}

to analyze KIT exons 9, 11,13 and 17 and PDGFRA exons $12,13,17$ and 18 as previously described (Willmore-Payne et al., 2005). The GIST tissue microarray (DAA1, SuperBioChips Laboratories) contained 48 GIST and 9 normal adult gastrointestinal tissue samples. The microarray GIST samples were KIT-positive by immunodetection, but their KIT mutation status was unknown.

\section{RNA isolation and quantitative RT-PCR}

Total RNA was extracted from paraffin-embedded GIST samples using the High Pure RNA Paraffin kit (Roche Diagnostic). For quantitative RT-PCR analysis, gene expression levels were measured using the LightCycler technology (Roche Diagnostics). PCR primers (Table 1) were designed using the LightCycler Probe Design software 2.0. Each sample was assayed in three independent experiments in triplicate. Expression levels were determined with the LightCycler analysis software (version 3.5) relative to standard curves. Data were represented as the mean level of gene expression relative to the expression of the reference gene HPRT (Roche Diagnostic). For cell culture experiments, RNA isolation was done with the RNeasy Mini kit (Qiagen) and quantitative RT-PCR was performed using Power Sybergreen (Applied biosystems). Data were represented as the mean level of gene expression relative to the expression of the reference genes $A B L$, TTC1 and $U B C$ for the comparison of RBPMS2 expression between different cell lines and GAPDH and ACTIN for GIST882 cells, which were treated with either Imatinib or U0126.

\section{Production of anti-RBPMS2 antibodies and cell culture}

The anti-human RBPMS2 rabbit polyclonal antibody was raised using a synthetic peptide corresponding to the C-terminus of human RBPMS2 (SSDTTQQGWKYRQ) (Fig. 3A). Anti-RBPMS2 antibodies were purified using protein A-sepharose and were tested by ELISA (Biotem, France).
The human GIST cell line GIST882 was maintained in DMEM (GIBCO) supplemented with 10\% Fetal Bovine Serum (FBS), 2\% PenicillineStreptomycin (Lonza) and incubated with $1 \mu \mathrm{M}$ of Imatinib (inhibitor of KIT activity) (LC Laboratory) or $5 \mu \mathrm{M}$ of U0126 (MEK inhibitor) (Sigma-Aldrich), as previously described (Gromova et al., 2011). The human Embryonic Kidney 293 (HEK293) cell line was grown in DMEM supplemented with 10\% FBS and transfected, using JetPEiTM (Polyplus, France), with $5 \mu \mathrm{g}$ of a construct in which the full length human RBPMS2 cDNA was subcloned in the pCS2 vector with an in frame N-terminal HA tag and the CMV promoter. Cells were analyzed after $24 \mathrm{~h}$. For western blot analyses, cells were lysed and protein extracts $(20 \mu \mathrm{g})$ were separated on $10 \%$ polyacrylamide gels (Bio-Rad Laboratories), transferred onto nitrocellulose membranes (Amersham Hybond-ECL) and incubated with anti-RBPMS2 (home-made), antiTubulin (Abcam) and anti-HA (Santa Cruz Biotechnologies) primary antibodies overnight. After several washes, membranes were incubated with the relevant horseradish peroxidase-conjugated secondary antibodies (Perkin Elmer). Detection was performed by chemiluminescence (Santa Cruz Biotechnologies) on Kodak films. Tubulin expression served as a loading control.

\section{Immunohistochemistry and in situ hybridization}

Immunofluorescence and immunohistochemistry analysis of paraffin-embedded GIST sections were performed as described (Rouleau et al., 2009; Rouleau et al., 2012). Briefly, sections were deparaffinized with Histoclear (VWR, France) and rehydrated through a series of graded alcohols to PBS. For immunodetection, heat-induced antigen retrieval was carried out in $10 \mathrm{mM}$ sodium citrate solution ( $\mathrm{pH}$ 9.0). Endogenous peroxidases were inactivated by incubation in $3 \% \mathrm{H}_{2} \mathrm{O}_{2}$ (Sigma-Aldrich) for 30 minutes. Anti-RBPMS2 (home-made), -Ki67 (NeoMarker), - $\alpha$ SMA (Sigma-Aldrich), -CD34 (Clinisciences), -S100 (Clinisciences), -KIT (Zymed), -Desmin (Euromedex) and

Fig. 2. Clinicopathological features and RBPMS2 mRNA expression in the adult GIST cohort. (A) Results of immunohistochemical staining for KIT (CD117), TMEM16A, CD34, $\alpha$ SMA (smooth muscle actin), DESMIN and S100 ( $n=23$ GIST samples). (B and C) KIT (B) and PDGFRA (C) expression level by quantitative RT-PCR in GIST samples ( $n=20$ ) and in normal samples from different regions of the gastrointestinal tract as controls $(n=5)$. KIT and PDGFRA mut., GIST samples with a mutation in KIT or PDGFRA; GIST WT, GIST samples with wild type KIT or PDGFRA; NA, not available. (D) RBPMS2 transcript level was determined by quantitative RT-PCR in GIST ( $n=20$ ) and in non-tumor samples from different regions of the gastrointestinal tract ( $n=5$, control). GIST WT, GIST samples with wild type KIT or PDGFRA; KIT and PDGFRA mut., GIST samples with a mutation in KIT or PDGFRA. (E) Comparison of the RBPMS2 transcript levels in the GIST and non-tumoral samples described in A with the Mann-Whitney test $(p<0.001)$. (F) Mean expression level of RBPMS2, KIT and PDGFRA in GISTs that were classified based on their KIT and PDGFRA mutational status. (G) Mean expression level of RBPMS2, KIT and PDGFRA in GISTs that were classified according to the risk of aggressive behavior. 
A

\begin{tabular}{c|cccc} 
Immunohistochemistry & Positive, $\mathbf{n}(\%)$ & Negative, $\mathbf{n}(\%)$ & NA, $\mathbf{n}(\%)$ & Total, $\mathbf{n}$ \\
\hline KIT (CD117) & $22(96)$ & $1(4)$ & $0(0)$ & 23 \\
TMEM16A & $16(69.5)$ & $4(17.5)$ & $3(13)$ & 23 \\
CD34 & $15(65)$ & $4(17.5)$ & $4(17.5)$ & 23 \\
SMOOTH MUSCLE ACTIN & $9(39)$ & $8(35)$ & $6(26)$ & 23 \\
DESMIN & $2(9)$ & $12(52)$ & $9(39)$ & 23 \\
S100 & $7(30)$ & $10(44)$ & $6(26)$ & 23
\end{tabular}

\section{B}

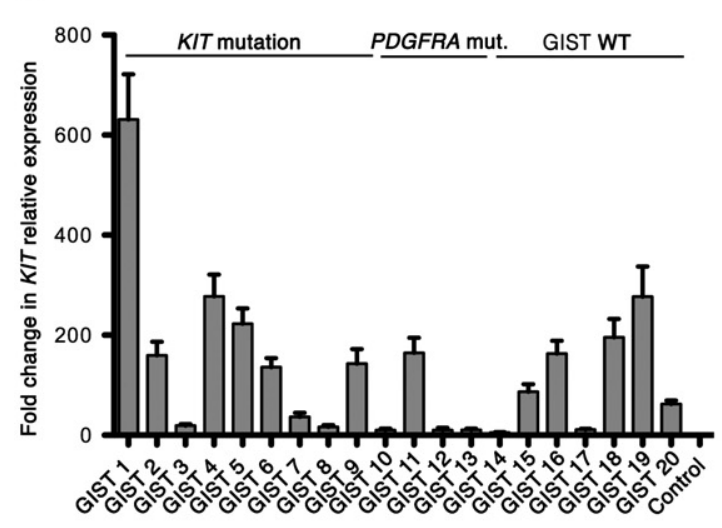

D

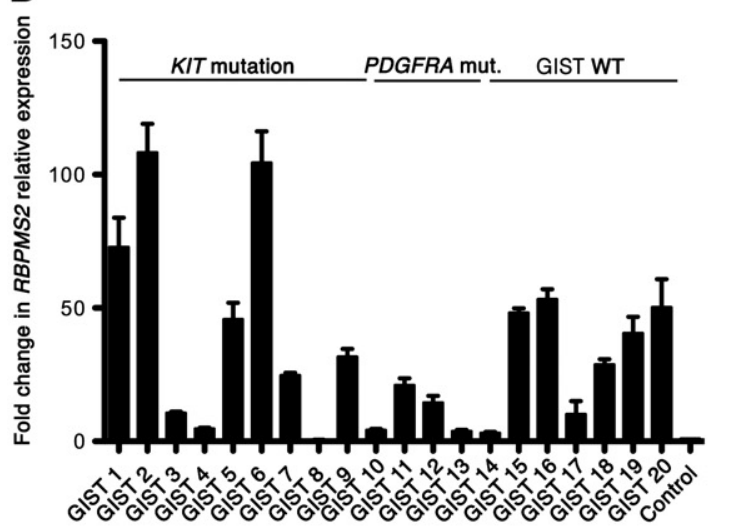

F

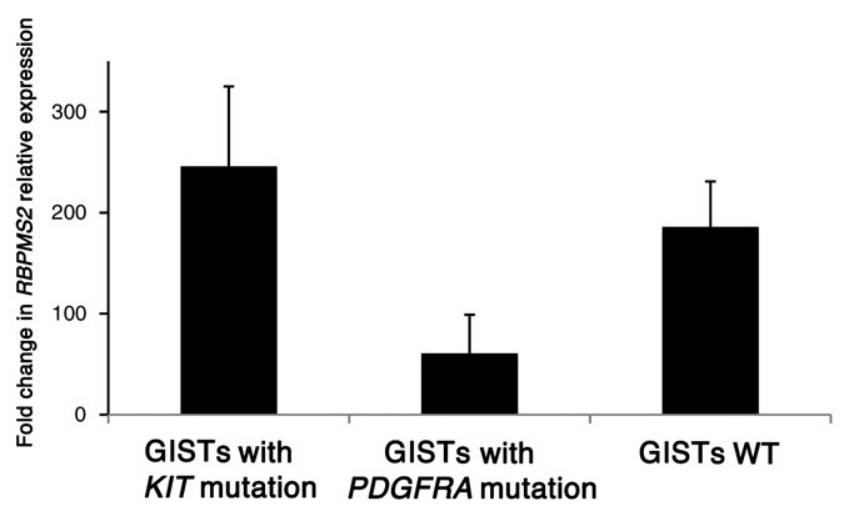

C

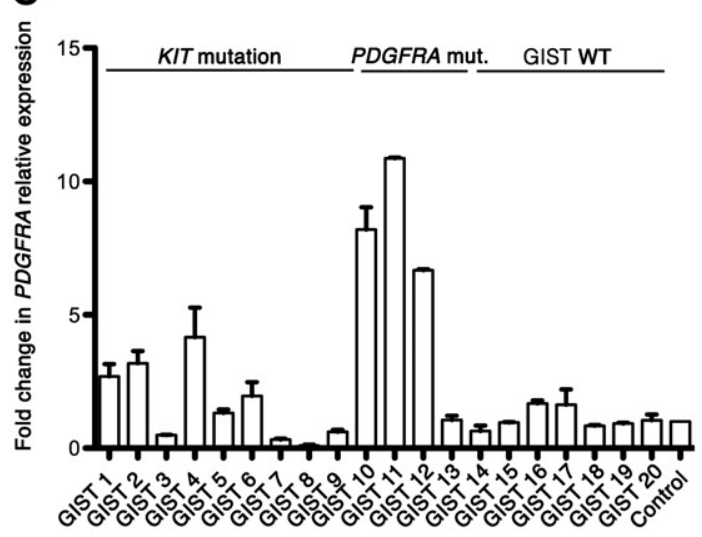

E

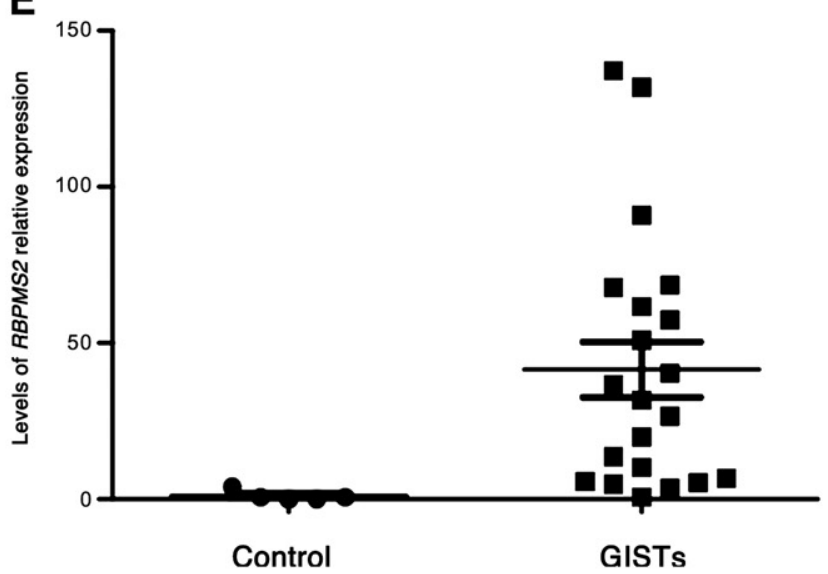

G

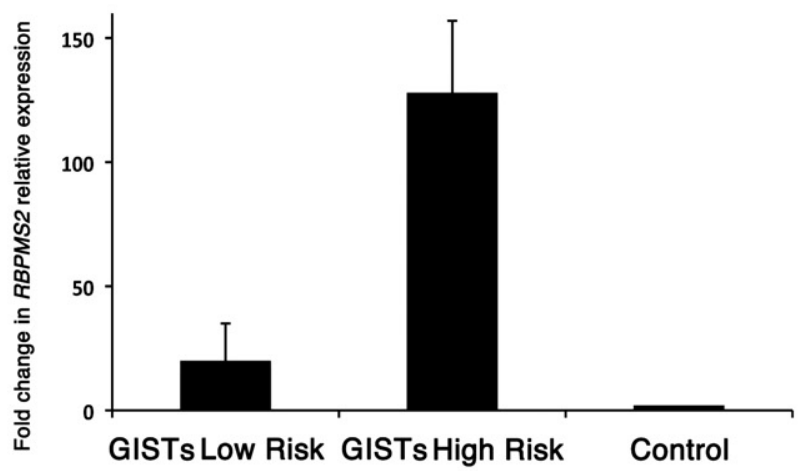


-TMEM16A (also called DOG1) (Abcam) primary antibodies were used. Specific mouse or rabbit anti-IgG biotinylated secondary antibodies were used with the avidin-peroxidase reagent (Vector) and antibody reactions were detected with 3,3'-Diaminobenzidine (SigmaAldrich). As control, each GIST sections were tested without primary antibodies. Hematoxylin and Eosin (H\&E) staining was performed

A

human RBPMS2 protein

MSNLKPDGEHGGSTGTGSGAGSGGALEEEVRTLFVSGLPVDIKPRELYLLFRPF KGYEGSLIKLTARQPVGFVIPDSRAGAEAAKNALNGIRFDPENPQTLRLEFAKA NTKMAKSKLMATPNPSNVHPALGAHFIARDPYDLMGAALIPASPEAWAPYPLY TTELTPAISHAAFTYPTATAAAAALHAQVRWYPSSDTTQQGWKYRQFC

B

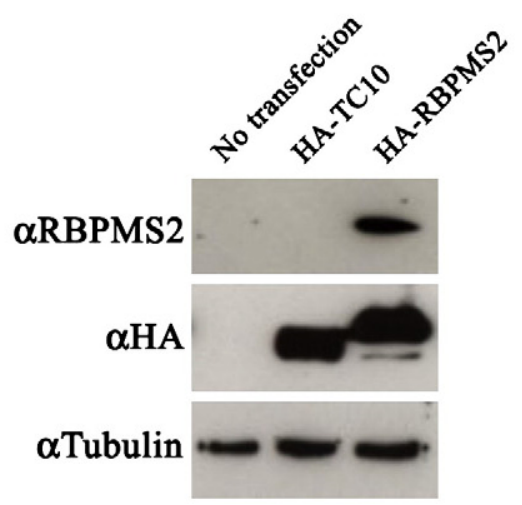

C

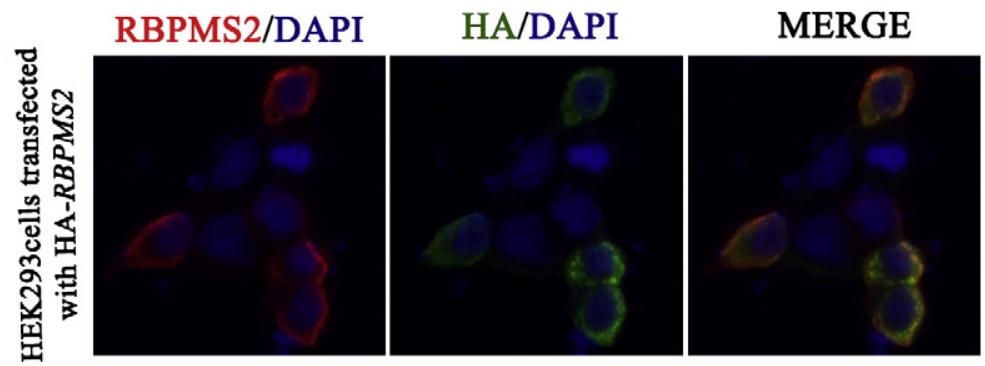

D
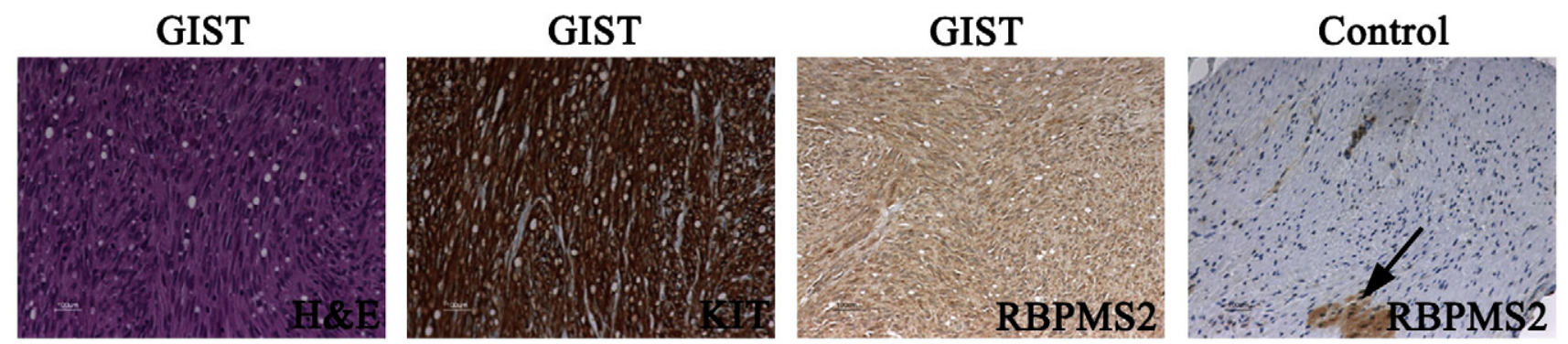

E

\begin{tabular}{c|cccc} 
Immunohistochemistry & Positive, n (\%) & Negative, n (\%) & NA, n (\%) & Total, $\mathbf{n}$ \\
\hline KIT (CD117) & $22(96)$ & $1(4)$ & $0(0)$ & 23 \\
RBPMS2 & $20(87)$ & $0(0)$ & $3(13)$ & 23
\end{tabular}

$\mathbf{F}$

GIST - Spindle cells

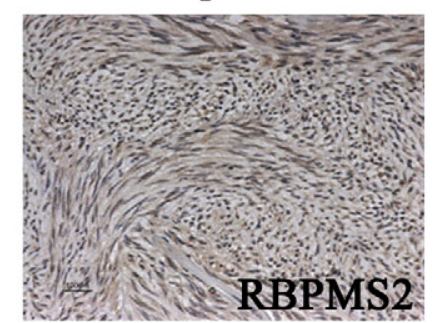

GIST - Mixed cells

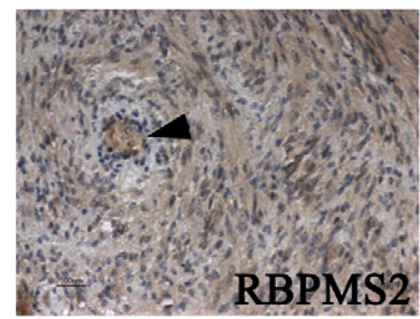

GIST - Epitheloid cells

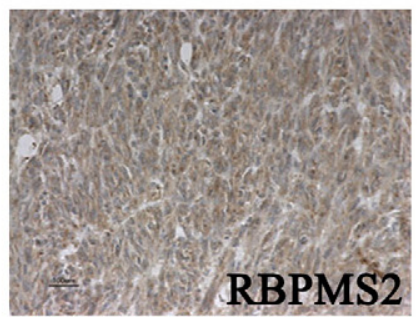


according to standard procedures. In situ hybridization experiments using paraffin sections were carried out as described (Come et al., 2006; Notarnicola et al., 2012). Anti-sense riboprobes were generated by PCR amplification of human RBPMS2 cDNA using specific primer sets and subcloned in pGEM T Easy Vector (Promega, France) as previously published (Notarnicola et al., 2012). Images were acquired using a Nikon-AZ100 stereomicroscope and a Carl-Zeiss Axiolmager microscope.

\section{Statistical analysis}

Statistical analysis was carried out using the Mann-Whitney test as previously described (Notarnicola et al., 2012).

\section{Results}

\section{RBPMS2 transcripts are detected in adult GISTs}

To determine whether the RNA-binding protein RBPMS2 was expressed in GISTs, we first analyzed by in situ hybridization a commercial tissue microarray that included 48 KIT-positive GISTs (from low to high risk) and 9 normal adult gastrointestinal tissues (Fig. 1). The level of RBPMS2 transcripts was very low in smooth muscles of normal adult gastrointestinal tissues (black arrows, Fig. 1). Conversely, RBPMS2 was strongly expressed in 36 of the 48 GIST samples (75\%), independently of their localization and the risk of aggressive behavior (red arrows, Fig. 1).

\section{Clinicopathological features of the GIST cohort}

Due to the limited clinical data and the absence of information on the KIT and PDGFRA mutational status for the GISTs included in the microarray and in order to better characterize RBPMS2 expression in GISTs, we analyzed GIST samples from a cohort of patients from Olomouc University Hospital. The group included 16 males and 7 females (male/female ratio: 1.44) with a mean age of 63.4 years (range: 36 to 81 years) who had exclusively primary GISTs located in the esophagus $(n=4)$, stomach $(n=5)$, small intestine $(n=8)$, rectum $(n=3)$, abdomen $(n=2)$ and soft tissues $(n=1)$ (Table 2$)$. All GISTs included in this study were characterized by using classical histopathological and immunohistochemical approaches with antiKIT, -SMA, -CD34, -Desmin, -S100 and - TMEM16A (DOG-1) antibodies (Fig. 2A; and data not shown). GISTs were divided in spindle ( $n=11)$, epithelioid $(n=4)$ and mixed cell (epithelioid and spindle) $(n=8)$ tumors based on the cell morphology (Table 2). Mitotic cells were detected with the anti-Ki67 antibody (summary in Fig. 2A) and tumors were classified as low risk $(n=10)$ or high risk $(n=13)$ as previously described (Miettinen and Lasota, 2006). KIT and PDGFRA mutational analysis was available for 21 GISTs and showed that 10 tumors (47.6\%) had KIT mutations (three GISTs with KIT mutations in exon 9, six tumors with mutations in exon 11 and one tumor with a mutation in exon 13), four (19\%) had a PDGFRA mutation (one missense and two silent mutations in exon 18 and one missense mutation in exon 12) and seven (33.4\%) had wild type (WT) KIT and PDGFRA (Table 2). Quantitative RT-PCR analysis of KIT and PDGFRA expression levels indicated, as previously published, that in all the analyzed GIST samples $(n=20)$ KIT and PDGFRA were up-regulated in comparison to normal gastrointestinal samples of adult colon, stomach and small intestine (Fig. 2B and C).

\section{RBPMS2 is strongly expressed in malignant GISTs}

We then analyzed the levels of RBPMS2 transcripts in this GIST cohort and in gastrointestinal control samples by quantitative RT-PCR. In most of the analyzed GIST samples (19 of 20) RBPMS2 expression level was higher than in control samples (Fig. 2D; Table 2) and the mean RBPMS2 level in GISTs was 42-fold higher than in control samples $(p<0.001)$ (Fig. 2E). The level of KIT and PDGFRA did not significantly correlated with the amount of RBPMS2 expression in the tumors (compare Fig. 2B and C and D; data not shown). Conversely, RBPMS2 expression was higher in GISTs harboring KIT mutations than in tumors with wild type KIT and with wild type KIT or PDGFRA mutations (Fig. 2F). GISTs with PDGFRA mutations were characterized by the lowest RBPMS2 expression levels (Fig. 2F). Finally, RBPMS2 levels were 64-fold higher in GIST samples with high risk of aggressive behavior than in adult control digestive samples (Fig. 2G) and 6.2-fold higher in high risk than in low risk GISTs (Fig. 2G).

\section{RBPMS2 protein is highly expressed in adult GISTs}

In order to examine RBPMS2 protein expression, we generated antibodies directed against the C-terminus of human RBPMS2 (Fig. 3A). The efficiency and the specificity of these anti-RBPMS2 antibodies were confirmed by western blot and immunofluorescence analyses in HEK293 cells that express HA tagged RBPMS2 (Fig. 3B, and C). In western blots, the anti-RBPMS2 antibody identified a single band of $27 \mathrm{kDa}$, corresponding to the predicted size of human RBPMS2 fused to the HA tag (Fig. 3B). In addition, this band was also detected with the anti-HA antibody (Fig. 3B). Moreover, the anti-RBPMS2- and - HA antibodies both detected an epitope localized in the cytoplasmic compartment of such cells (Fig. 3C). Finally, the RBPMS2 signal was specifically lost when the anti-RBPMS2 antibody was pre-incubated with the RBPMS2 C-terminal peptide used to produce the antibody (data not shown). We then examined the expression of RBPMS2 by immunohistochemistry in control gastrointestinal and GIST samples (Fig. 3D-F). RBPMS2 was faintly but reproductively detected in the gastrointestinal musculature with the exception of the myenteric plexus (arrow, Fig. 3D). In contrast, it was strongly detected in $87 \%$ of the analyzed GIST samples (Fig. 3D, representative GIST sample, and Fig. 3E) and its expression level was comparable in spindle, epithelioid and mixed cell tumor variants (Fig. 3F).

\section{RBPMS2 expression and regulation in GIST882 cells}

To position RBPMS2 in the KIT signaling pathway in GISTs, we first compared RBPMS2 expression in GIST882 (a GIST cell line homozygous for the oncogenic KIT mutation K641E with strong KIT expression and high level of KIT activity) (Tuveson et al., 2001), HeLa and 1321N1 (human astrocytoma) cells as well as in two prostate cancer cell lines (PC3 and LNCaP). RBPMS2 mRNA level was relatively high in

Fig. 3. RBPMS2 protein expression in adult GISTs. Characterization of the anti-human RBPMS2 antibody by immunofluorescence and western blotting (A-C). (A) Sequence of the human RBPMS2 protein (amino acids 1-209). Amino acids 195-207 of the C-terminus (in red) correspond to the sequence of the synthesized peptide used for producing the anti-RBPMS2 antibody. (B) Western blot analysis of HEK293 cells following transfection of empty vector (lane 1), the HA-TC10 (lane 2) or the HA-RBPMS2 construct (lane 3). $20 \mu \mathrm{g}$ of whole protein extracts were processed for each condition. Tubulin (50 kDa) was used as loading protein control. HA-RBPMS2 was detected as a $27 \mathrm{kDa}$ band with both anti-HA and anti-RBPMS2 antibodies. In addition, lower band corresponding to a C-terminal product of degradation was observed with only anti-HA antibodies. (C) HEK293 cells were transfected with the HA-RBPMS2 construct and then processed for immunofluorescence analysis using mouse anti-HA and anti-RBPMS2 primary antibodies and anti-mouse Alexa488 and anti-rabbit Alexa555 IgG, respectively. Nuclei were stained with Hoechst. Cytoplasmic localization of the signal is observed with both antibodies. (D) Representative example of RBPMS2 protein expression in a colonic, high risk GIST sample. Histological staining (H\&E) and labeling with anti-KIT (CD117) and anti-RBPMS2 antibodies. In normal colon (control), RBPMS2 is expressed in vessels and the enteric myenteric plexus (arrow), but not in visceral SMCs. (E) Summary of KIT (CD117) and RBPMS2 protein expression in the GIST cohort $(n=23)$. NA, not available. (F) Analysis of the expression of RBPMS2 in the GIST samples that were classified based on their morphological features (spindle, mixed and epithelioid cells). Arrowhead shows vessels. 
A

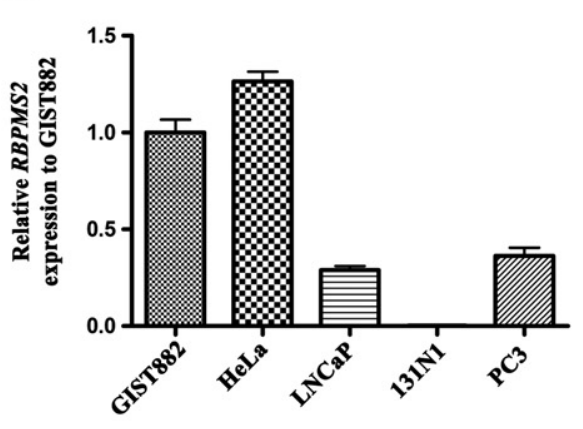

B

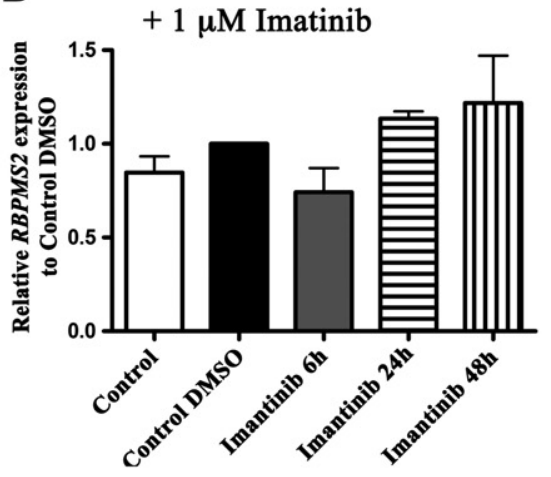

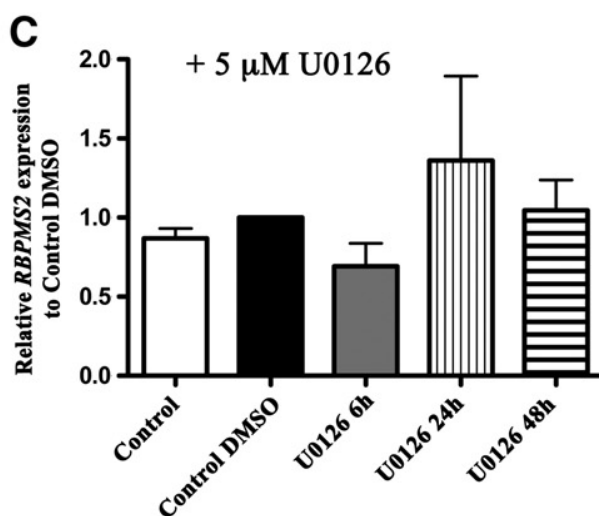

Fig. 4. RBPMS2 mRNA expression and regulation in the GIST882 cell line. (A) Analysis of RBPMS2 expression by quantitative RT-PCR in GIST882, HeLa, LNCaP, 1321 N1 and PC3 cells. RBPMS2 is strongly expressed in GIST882 and HeLa cells, weakly in LNCaP and PC3 prostate cancer cells and undetectable in 1321 N1 astrocytoma cells. (B) Analysis of RBPMS2 transcript level in GIST882 cells upon incubation with $1 \mu \mathrm{M}$ of Imatinib (inhibitor of KIT activity) or with DMSO alone (control DMSO) for 6, 24 and 48 h. RBPMS2 transcript level is not influenced by inhibition of KIT activity. Control, untreated cells. (C) Analysis of RBPMS2 transcript level in GIST881 cells following incubation with $5 \mu$ M of U0126 (inhibitor of MEK activity) or with DMSO alone (control DMSO) for 6, 24 and 48 h. RBPMS2 transcript level is not influenced by MEK inhibition. Control, untreated cells.

GIST882 and HeLa cells in comparison to 1321N1 cells and the two prostate cancer cell lines (Fig. 4A). Then GIST882 cells were treated with $1 \mu \mathrm{M}$ of Imatinib (a specific inhibitor of KIT activity) (Tuveson et al., 2001) or with $5 \mu \mathrm{M}$ of U0126 (a selective inhibitor of MEK which is a downstream effector of the KIT signaling pathway) for 6 , 24 and $48 \mathrm{~h}$ (Fig. 4B, and C) and RBPMS2 expression was determined by quantitative PCR. No significant changes in RBPMS2 mRNA levels were observed following addition of Imatinib or U0126 in the culture medium (Fig. 4B and C).

\section{Discussion}

In this study we analyzed the expression of the RNA-binding protein RBPMS2 in a cohort of GIST samples that were classified according to their KIT/PDGFRA mutational status, risk of aggressive behavior and histological pattern (spindle, epithelioid and mixed cell phenotype).

We found that RBPMS2 mRNA and protein expression was significantly higher in GIST samples than in control gastrointestinal tissues, particularly in high-risk tumors. The levels of KIT and PDGFRA did not significantly correlate with the amount of RBPMS2 expression. However, RBPMS2 mRNA levels were higher in GISTs harboring KIT mutations than in tumors with PDGRA mutations or with wild type KIT and PDGFRA. This difference could be due to the different origins of KIT and PDGFRA expressing cells and would suggest that RBPMS2 and KIT expression are linked. Moreover, in the GIST882 cell line that carries the KIT mutation K641E and shows high KIT activity, inhibition of KIT activity using Imatinib treatment did not alter the expression of RBPMS2. In contrast, using primary embryonic digestive smooth muscle cultures we found that RBPMS2 over-expression induces a 2-fold increase of KIT mRNA level (Notarnicola et al., 2012; Notarnicola and de Santa Barbara, unpublished data). All these data suggest that KIT and RBPMS2 could be expressed in the same mesenchymal cells during the early development and remodeling of mesenchymal-derived gastrointestinal cells, or that they are part of the same signaling pathway, or share a regulatory circuitry. More experiments are needed to confirm these hypotheses and to position RBPMS2 relative to the KIT signaling pathway.

Chromosomal alterations during GIST progression have been described and could be involved in GIST prognosis (Corless et al., 2011). Specifically, losses and gains on chromosome 15 between $15 q 22.1$ and $15 q 25.3$ have been clearly correlated with poor outcome (Ylipää et al., 2010). As the RBPMS2 gene is localized on chromosome $15 q 22.31$, we can hypothesize based on our results that elimination of a negative regulatory sequence necessary for correct RBPMS2 expression could alter the endogenous expression of RBPMS2, thus favoring dedifferentiation of mesenchymal cells that will give rise to GIST tumors. A thorough characterization of the correlation between alterations of this genome region and RBPMS2 expression is now required.

In conclusion, our study shows that most of the analyzed GISTs are characterized by abnormally elevated expression of RBPMS2, suggesting that RBPMS2 could be an indicator for tumor progression and a potential target for cancer therapy in GIST.

\section{Conflict of interest statement}

The authors declare that there are no conflicts of interest.

\section{Contribution to authorship}

Immunohistochemistry and in situ hybridization were carried out by $\mathrm{IH}$ with the help of $\mathrm{CR}, \mathrm{FB}$ and $\mathrm{SF}$ under the supervision of PdSB. GIST collection and analyses were carried out by JS, MJ, and IH. Quantitative PCR analysis was performed by IH with the help of $\mathrm{CN}$, and MR under the supervision of PdSB and JV. GIST cell line analyses were carried out by AT under the supervision of JMV. PdSB wrote the paper.

\section{Funding}

The work was supported by ARC (Association pour la Recherche sur le Cancer), Région Languedoc-Roussillon (Chercheur d'Avenir), INCA GSO Emergence and Ligue contre le Cancer (Comité de l'Aude) to PdSB. IH was supported by a studentship of the French Ministry of Foreign Affairs and by an IGA studentship of Palacky University, Olomouc. AT is $\mathrm{PhD}$ student bursary on Télévie (Belgium) grant 7.4562.10. JMV is Research Director at FRS-FNRS (Belgium).

\section{Acknowledgments}

We thank members of INSERM U1046 for helpful discussions.

\section{References}

Come, C., Magnino, F., Bibeau, F., et al., 2006. Snail and slug play distinct roles during breast carcinoma progression. Clinical Cancer Research 12, 5395-5402.

Corless, C.L., Barnett, C.M., Heinrich, M.C., 2011. Gastrointestinal stromal tumours: origin and molecular oncology. Nature Reviews. Cancer 11, 865-878.

Delahaye, N.F., Rusakiewicz, S., Martins, I., et al., 2011. Alternatively spliced NKp30 isoforms affect the prognosis of gastrointestinal stromal tumors. Nature Medicine 17, 700-707.

Faure, S., de Santa Barbara, P., 2011. Molecular embryology of the foregut. Journal of Pediatric Gastroenterology and Nutrition 52, S2-S3.

Fletcher, C.D., Berman, J.J., Corless, C., et al., 2002. Diagnosis of gastrointestinal stromal tumors: a consensus approach. Human Pathology 33, 459-465. 
Gromova, P., Rubin, B.P., Thys, A., et al., 2011. Neurotensin receptor 1 is expressed in gastrointestinal stromal tumors but not in interstitial cells of Cajal. PLoS One 6 , e14710.

Hirota, S., Isozaki, K., Moriyama, Y., et al., 1998. Gain-of-function mutations of c-kit in human gastrointestinal stromal tumors. Science 279, 577-580.

Joensuu, H., Roberts, P.J., Sarlomo-Rikala, M., et al., 2001. Effect of the tyrosine kinase inhibitor STI571 in a patient with a metastatic gastrointestinal stromal tumor. The New England Journal of Medicine 344, 1052-1056.

Le Guen, L., Notarnicola, C., de Santa Barbara, P., 2009. Intermuscular tendons are essential for the development of vertebrate stomach. Development 136, 791-801.

Miettinen, M., Lasota, J., 2006. Gastrointestinal stromal tumors: review on morphology, molecular pathology, prognosis, and differential diagnosis. Archives of Pathology \& Laboratory Medicine 130, 1466-1478.

Notarnicola, C., Rouleau, C., Le Guen, L., et al., 2012. The RNA binding protein RBPMS2 regulates gastrointestinal smooth muscle development. Gastroenterology 143, 687-697.

Renouf, D.J., Wilson, L., Blanke, C.D., 2009. Successes and challenges in translational research: the development of targeted therapy for gastrointestinal stromal tumours. Clinical Cancer Research 15, 3908-3911.

Rouleau, C., Matecki, S., Kalfa, N., et al., 2009. Activation of MAP kinase (ERK1/2) in human neonatal colonic enteric nervous system. Neurogastroenterology and Motility $21,207-214$.
Rouleau, C., Rico, C., Hapkova, I., et al., 2012. Immunohistochemical analysis of bone morphological protein signaling pathway in human myometrium. Experimenta and Molecular Pathology 93, 56-60.

Sanders, K.M., Koh, S.D., Ward, S.M., 2006. Interstitial cells of Cajal as pacemakers in the gastrointestinal tract. Annual Review of Physiology 68, 307-343.

St Johnston, D., 2005. Moving messages: the intracellular localization of mRNAs. Nature Reviews Molecular Cell Biology 6, 363-375.

Tuveson, D.A., Willis, N.A., Jacks, T., et al., 2001. STI571 inactivation of the gastrointestinal stromal tumor c-KIT oncoprotein: biological and clinical implications. Oncogene 20, 5054-5058.

van Kouwenhove, M., Kedde, M., Agami, R., 2011. MicroRNA regulation by RNA-binding proteins and its implications for cancer. Nature Reviews. Cancer 11, 644-656.

Willmore-Payne, C., Layfield, L.J., Holden, J.A., 2005. C-KIT mutation analysis for diagnostic of gastrointestinal stromal tumors in fine needle aspiration specimens. Cancer 105 $165-170$.

Xin, M., Small, E.M., Sutherland, L.B., et al., 2009. MicroRNAs miR-143 and miR-145 modulate cytoskeletal dynamics and responsiveness of smooth muscle cells to injury. Genes \& Development 23, 2166-2178.

Ylipää, A., Hunt, K.K., Yang, J., et al., 2010. Integrative genomic characterization and genomic staging system for gastrointestinal stromal tumors. Cancer 117, 380-389. 